\title{
Chemical Quality of Depositional Sediments and Associated Soils in New Orleans and the Louisiana Peninsula following Hurricane Katrina
}

Craig Adams ${ }^{1 *}$, Emitt C. Witt ${ }^{2}$, Jianmin Wang ${ }^{1}$, David K. Shaver ${ }^{2}$,David Summers ${ }^{3}$, Youssef Filali-Meknassi ${ }^{1}$, Honglan Shi ${ }^{1}$, Ronaldo Luna ${ }^{1}$, Neil Anderson ${ }^{4}$

\footnotetext{
${ }^{1}$ University of Missouri-Rolla, Environmental Research Center for Emerging Contaminants, Rolla, MO 65409; ${ }^{2}$ United States Geological Survey, Mid-Continent Geographic Science Center, Rolla, MO 65409; ${ }^{3}$ University of Missouri-Rolla, Rock Mechanics and Explosives Center, Rolla, MO 65409; ${ }^{4}$ University of Missouri-Rolla, Natural Hazard Mitigation Institute, Rolla, MO 65409; ${ }^{*}$ Corresponding Author
}

CORRESPONDING AUTHOR FOOTNOTE: University of Missouri-Rolla, 220 Butler-Carlton Hall, Rolla, MO 65409, TL: 573-341-4041, FX: 573-341-7219, adams@umr.edu

Manuscript for Environmental Science and Technology 


\section{SI Table 1: Results of organic chemical analysis for sediment samples collected in New Orleans and the Louisiana peninsula.}

\begin{tabular}{|c|c|c|c|c|c|c|c|c|c|c|c|c|c|c|c|c|c|c|c|c|c|}
\hline \multirow[b]{2}{*}{ Site } & \multirow[b]{2}{*}{ Latitude } & \multirow[b]{2}{*}{ Longitude } & \multirow[b]{2}{*}{ 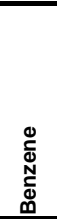 } & \multicolumn{14}{|c|}{ Pesticide $(\mu \mathrm{g} / \mathrm{kg})$} & \multirow[b]{2}{*}{ 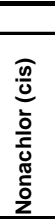 } & \multirow[b]{2}{*}{ 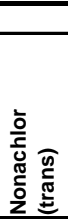 } & \multirow[b]{2}{*}{ 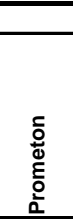 } & \multirow[b]{2}{*}{ 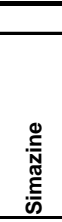 } \\
\hline & & & & $\begin{array}{l}\text { 흔 } \\
\frac{1}{0} \\
\frac{0}{8} \\
\\
\end{array}$ & $\begin{array}{l}\frac{1}{} \\
\frac{1}{0} \\
\frac{\pi}{4} \\
\end{array}$ & $\frac{\mathfrak{c}}{\frac{3}{2}}$ & 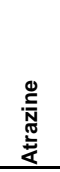 & 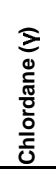 & 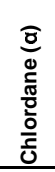 & 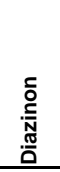 & 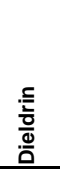 & 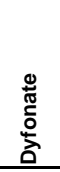 & 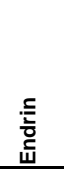 & 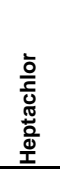 & 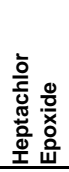 & 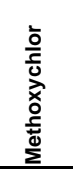 & $\begin{array}{l}\frac{1}{0} \\
\frac{1}{0} \\
\frac{\pi}{0} \\
\frac{0}{0}\end{array}$ & & & & \\
\hline 1 & 30.02936 & -90.21708 & $\mathrm{D}$ & ND & ND & ND & ND & ND & ND & ND & ND & ND & ND & ND & ND & ND & ND & ND & ND & ND & ND \\
\hline 2 & 30.02936 & -90.21708 & $\ldots$ & $\ldots$ & $-\cdots$ &.- & $\ldots$ & $-\ldots$ & $\ldots$ & - & $\ldots$ & -- & $\ldots$ & $-\ldots$ & $-\cdots$ &.- & $-\cdots$ & $\ldots$ & -- & - & $-\ldots$ \\
\hline 3 & 30.02072 & -90.20486 & $\mathrm{D}$ & $\ldots$ & $\ldots$ & $\ldots$ & $\ldots$ & $\ldots$ & $\ldots$ & $\ldots$ & $\ldots$ & $\ldots$ & - - - & - - - & $\ldots$ & $\ldots$ & $\ldots$ & $\ldots$ & $\ldots$ & - - - & $\ldots$ \\
\hline 4 & 30.01617 & -90.19808 & $\mathrm{D}$ & $\ldots$ & $\ldots$ & $\ldots$ & $\ldots$ & $\ldots$ & $\ldots$ & $\ldots$ & $\ldots$ & $\ldots$ & $\ldots$ & $\ldots$ & $\ldots$ & $\ldots$ & $\ldots$ & $\ldots$ & $\ldots$ & $\ldots$ & $\ldots$ \\
\hline 5 & 30.01528 & -90.16311 & $\mathrm{D}$ & $\ldots$ & $\ldots$ & $\ldots$ & $\ldots$ & $\ldots$ & $\ldots$ & $\ldots$ & $\ldots$ & $\ldots$ & $\ldots$ & $\ldots$ & $\ldots$ & $\ldots$ & $\ldots$ & $\ldots$ & $\ldots$ & $\ldots$ & $\ldots$ \\
\hline 6 & 30.01782 & -90.12039 & D & ND & ND & ND & ND & ND & ND & ND & ND & ND & ND & ND & ND & 425 & ND & ND & ND & ND & ND \\
\hline 7 & 30.00392 & -90.11400 & $\mathrm{D}$ & ND & ND & ND & ND & ND & ND & ND & ND & ND & ND & ND & ND & 130 & ND & ND & ND & ND & ND \\
\hline 8 & 30.00392 & -90.11400 &.- & ND & 185 & ND & ND & ND & ND & 152 & ND & 66 & ND & ND & 224 & 496 & ND & ND & ND & ND & ND \\
\hline 9 & 30.02558 & -90.02314 & $\mathrm{D}$ & $\ldots$ & - . & $\ldots$ & - . & $\ldots$ & $\ldots$ & $\ldots$ & $\ldots$ & $\ldots$ & - - & - . & $\ldots$ & $\ldots$ & $\ldots$ & $\ldots$ & $\ldots$ & - - & $\ldots$ \\
\hline 10 & 30.02858 & -90.01414 & $\mathrm{D}$ & ND & 337 & ND & ND & ND & ND & 306 & ND & 12 & ND & 47 & 10 & 206 & ND & ND & ND & 36 & ND \\
\hline 11 & 30.03011 & -89.99758 & ND & ND & ND & ND & ND & 61 & ND & ND & ND & ND & ND & 24 & ND & ND & ND & 32 & 67 & ND & ND \\
\hline 12 & 30.04906 & -89.98042 & D & ND & ND & ND & ND & ND & ND & ND & ND & ND & ND & ND & ND & ND & ND & ND & ND & ND & ND \\
\hline 13 & 30.04664 & -89.97233 & $\ldots$ & $\ldots$ & $\ldots$ & $\ldots$ & $\ldots$ & $\ldots$ & $\ldots$ & $\ldots$ & $\ldots$ & $\ldots$ & - - & - . & $\ldots$ &.- &.- & $\ldots$ &.- & $\ldots$ & - . \\
\hline 14 & 30.04461 & -89.96014 & $\mathrm{D}$ & ND & ND & ND & ND & ND & ND & 168 & ND & ND & ND & 189 & ND & 309 & ND & ND & ND & ND & ND \\
\hline 15 & 29.95125 & -90.11003 &.- & ND & $\ldots$ & $\ldots$ & - . & ND & ND & ND & $\ldots$ & 14 & ND & - . & ND & 76 & ND & $\ldots$ & $\ldots$ & ND & \\
\hline 16 & 29.94629 & -90.10763 & $\ldots$ & ND & ND & ND & ND & ND & ND & ND & 15 & ND & ND & ND & ND & 347 & ND & ND & 63 & ND & ND \\
\hline 17 & 29.94123 & -90.10339 & $\ldots$ & ND & $\ldots$ & $\ldots$ & $\ldots$ & ND & ND & ND & $\ldots$ & ND & ND & $\ldots$ & ND & 135 & ND & $\ldots$ &.- & ND & $\ldots$ \\
\hline 18 & 29.94235 & -90.09671 & $\mathrm{D}$ & 175 & ND & ND & ND & ND & ND & ND & ND & ND & ND & ND & 26 & 3359 & ND & ND & ND & ND & ND \\
\hline 19 & 29.9 & -90.0 & $\ldots$ & 153 & ND & ND & ND & ND & ND & 180 & ND & ND & ND & ND & ND & ND & ND & ND & ND & ND & ND \\
\hline 20 & 29.96911 & -90.02106 & $\cdots$ & 151 & ND & ND & ND & ND & ND & - - & ND & 11 & ND & ND & ND & ND & ND & ND & ND & ND & ND \\
\hline 21 & 29.96858 & -90.01967 & ND & 157 & ND & ND & ND & ND & ND & ND & ND & ND & ND & ND & ND & ND & ND & ND & ND & ND & ND \\
\hline 22 & 29.95058 & -89.95986 & $\ldots$ & ND & ND & ND & 66 & ND & ND & ND & ND & ND & ND & 150 & ND & 141 & ND & ND & ND & ND & ND \\
\hline 23 & 29.9 & -89.95986 & $\mathrm{D}$ & $\ldots$ &.- & $\ldots$ & - - & $\ldots$ & $\ldots$ & - . & - . & $\ldots$ & - - & - - & - - & - . & $\ldots$ & $\ldots$ & - - & - . & - . \\
\hline 24 & 29.93364 & -89.96783 & ND & ND & ND & ND & ND & ND & ND & ND & ND & ND & ND & ND & ND & 379 & ND & ND & ND & ND & ND \\
\hline 25 & 29.93364 & -89.96783 & $\mathrm{D}$ & $\ldots$ &.- & $\ldots$ & $\ldots$ & - . & $\ldots$ &.- & $\ldots$ & - - & - - & $\ldots$ & $\ldots$ & $\ldots$ & $\ldots$ & $\ldots$ & - - & - - & - - \\
\hline 26 & 29.9 & & D & ND & 53 & ND & ND & ND & ND & ND & ND & ND & ND & ND & ND & 200 & ND & ND & ND & ND & ND \\
\hline 27 & 29.93800 & -89.93558 & $\mathrm{D}$ & - - & -- & - - & - - & - - & - - & - - & $\cdots$ & -- & -- & - - & - - & - - & - - & $\cdots$ & $-\cdots$ & $-\cdots$ & - - \\
\hline 28 & 29.93800 & -89.93558 & $\ldots$ & 146 & ND & ND & ND & ND & ND & ND & ND & ND & ND & ND & ND & ND & ND & ND & ND & ND & ND \\
\hline 29 & 29.93414 & -89.94444 & $\mathrm{D}$ & ND & 54 & ND & 796 & ND & ND & 401 & 431 & 17 & ND & ND & ND & 937 & ND & ND & ND & 475 & 3128 \\
\hline 30 & 29.93414 & -89.94444 & D & ND & 70 & ND & ND & ND & ND & 300 & ND & 13 & ND & 103 & ND & ND & ND & ND & ND & 198 & 190 \\
\hline 31 & 29.93414 & -89.94444 & $\mathrm{D}$ & $\ldots$ & - - & $\ldots$ & - - & - . & - . & $\ldots$ & $\ldots$ & - . & - - & - . & $\ldots$ & $\ldots$ & $\ldots$ & $\ldots$ & - - & - . & - . \\
\hline 32 & 29.93139 & -89.94589 & $\mathrm{D}$ & ND & 81 & ND & 731 & ND & ND & ND & ND & 49 & ND & 75 & ND & 649 & ND & ND & ND & 645 & 1308 \\
\hline 33 & 29.93139 & -89.94589 & ND & 150 & 50 & 362 & 552 & ND & ND & 536 & ND & 137 & ND & ND & ND & ND & ND & ND & ND & 749 & 737 \\
\hline 34 & 29.93164 & -89.93672 & $\ldots$ & - . & - - & - . & - - & $\ldots$ & $\ldots$ & - . & $\ldots$ & - - & - - & - . & - . & - . & $\ldots$ & $\ldots$ & - - & - - & $\ldots$ \\
\hline 35 & 29.93164 & -89.93672 & $\mathrm{D}$ & 157 & ND & ND & ND & ND & ND & ND & ND & ND & ND & ND & ND & 37 & ND & ND & 14 & ND & ND \\
\hline 36 & 29.67031 & -89.97228 & ND & ND & ND & ND & ND & ND & ND & ND & ND & ND & ND & 204 & ND & ND & ND & ND & ND & ND & ND \\
\hline 37 & 29.50036 & -89.72013 & $\ldots$ & ND & ND & ND & ND & ND & 109 & ND & ND & 16 & ND & ND & 277 & 71 & ND & ND & 11 & ND & ND \\
\hline 38 & 29.47453 & -89.68794 & $\mathrm{D}$ & 241 & ND & ND & ND & ND & ND & 200 & ND & ND & ND & ND & ND & 1306 & ND & ND & ND & ND & 635 \\
\hline 39 & 29.47217 & -89.68544 & $\ldots$ & $\ldots$ & ND & ND & ND & ND & ND & - . & ND & ND & ND & ND & ND & 830 & $\ldots$ & ND & ND & 16 & ND \\
\hline 40 & 29.45642 & -89.66294 & $\mathrm{D}$ & ND & 92 & ND & ND & ND & ND & ND & ND & ND & ND & ND & ND & ND & ND & ND & ND & ND & ND \\
\hline 41 & 29.38832 & -89.60504 & ND & 402 & 180 & ND & 742 & ND & ND & 423 & ND & 37 & ND & ND & ND & 909 & ND & ND & ND & 2581 & ND \\
\hline 42 & 29.33595 & -89.49522 & $\mathrm{D}$ & ND & ND & ND & ND & ND & ND & ND & ND & ND & ND & ND & ND & ND & ND & ND & ND & ND & ND \\
\hline 43 & 29.35081 & -89.45961 & $\mathrm{D}$ & ND & 251 & ND & ND & ND & ND & 176 & ND & 33 & ND & 101 & 30 & 213 & ND & ND & ND & ND & ND \\
\hline 44 & 29.30897 & -89.36636 & $\ldots$ & ND & ND & ND & ND & ND & ND & ND & ND & 14 & ND & 33 & ND & ND & ND & ND & ND & ND & ND \\
\hline 45 & 29.27131 & -89.35386 & $\mathrm{D}$ & ND & ND & ND & ND & ND & ND & ND & ND & ND & ND & ND & ND & 194 & ND & ND & ND & ND & ND \\
\hline 46 & 29.23153 & -89.39014 & ND & ND & 113 & ND & ND & ND & ND & 162 & ND & ND & ND & ND & ND & 114 & ND & ND & ND & ND & 56 \\
\hline
\end{tabular}

